\title{
ON THE CHARACTERISATION OF ASPLUND SPACES
}

\author{
J. R. GILES \\ (Received 16 May 1980; revised 19 November 1980) \\ Communicated by J. B. Miller
}

\begin{abstract}
A Banach space is an Asplund space if every continuous convex function on an open convex subset is Fréchet differentiable on a dense $G_{\delta}$ subset of its domain. The recent research on the RadonNikodým property in Banach spaces has revealed that a Banach space is an Asplund space if and only if every separable subspace has separable dual. It would appear that there is a case for providing a more direct proof of this characterisation.
\end{abstract}

1980 Mathematics subject classification (Amer. Math. Soc.): 46 B 20, 46 B 22.

\section{Introduction}

A continuous convex function on an open interval of the real numbers is differentiable everywhere except at most on a countable subset of its domain. There has been interest in the problem of characterising those Banach spaces where the continuous functions exhibit similar differentiability properties.

The earliest result in this line was given by Mazur (1933) who showed that in a separable Banach space every continuous convex function on an open convex subset is Gâteaux differentiable on a dense $G_{\delta}$ subset of its domain.

Contemporary interest in the problem was renewed by Asplund (1968) who showed that in a Banach space with separable dual every continuous convex function on an open convex subset is Fréchet differentiable on a dense $G_{\delta}$ subset of its domain.

We say that a Banach space is an Asplund space if every continuous convex function on an open convex subset is Fréchet differentiable on a dense $G_{\delta}$ subset of its domain.

(1) Copyright Australian Mathematical Society 1982 
During the 1970's there was intensive study of geometric equivalents of the Radon-Nikodým property in Banach spaces. An important development in this study was the discovery by Namioka and Phelps (1975) that the dual of an Asplund space has the Radon-Nikodým property. Stegall (1978) established the full duality: a Banach space is an Asplund space if and only if the dual space has the Radon-Nikodým property.

So then Stegall's (1975) satisfying characterisation of Banach spaces whose dual has the Radon-Nikodým property provided the following characterisation of Asplund spaces.

THEOREM. A Banach space is an Asplund space if and only if every separable subspace has separable dual.

It was clear from the beginning that Mazur's direct proof was not capable of being generalised to the Fréchet differentiability case. Asplund's proof relied on renorming theorems and on the use of Fenchel duality of convex functions. The proof of the final characterisation theorem has been heavily influenced by geometry of the dual space developed initially to deal with the Radon-Nikodým property in the dual.

As it now stands the most recent version of the proof as given by Phelps (1978) depends on an ingenious lemma (Lemma 2.2, Phelps (1978)) which on the face of it has little natural motivation from the problem itself. Furthermore, the proof still depends heavily on dual methods. It would appear that there is a case for providing a more direct proof of the characterisation theorem.

The proof we offer falls into two parts. We prove

(i) a separable Banach space is Asplund if and only if it has separable dual, and then

(ii) for a Banach space the Asplund property is separably determined; that is, a Banach space is Asplund if and only if every separable closed subspace is Asplund.

Ekeland and Lebourg (1976) provided a very general condition which is sufficient for a Banach space to be Asplund. We conclude with a simplified version of this proof.

\section{The characterisation theorem for the separable case}

Consider a continuous convex function $\phi$ on an open convex subset $A$ of a real normed linear space $(X,\|\cdot\|)$ with dual space $X^{*}$. Given $x \in A$, the set

$$
\partial \phi(x) \equiv\left\{f \in X^{*}: f(y-x)<\phi(y)-\phi(x) \text { for all } y \in A\right\}
$$

of subgradients of $\phi$ at $x$ is a non-empty weak* compact convex subset of $X^{*}$. 
It is well known (see Phelps (1978)) that the subgradient mapping $x \mapsto \partial \phi(x)$ of $A$ into subsets of $X^{*}$ is locally bounded; that is, given $a \in A$ there exists a $K>0$ and a $\delta(a)>0$ such that $\|f\|<K$ for all $f \in \partial \phi(x)$ and $\|x-a\|<\delta$, and weak* upper semi-continuous; that is, given $a \in A$ and weak* open set $W \supseteq \partial \phi(a)$ there exists a $\delta(a)>0$ such that $\partial \phi(x) \subseteq W$ for all $\|x-a\|<\delta$.

It is useful for our purposes to introduce an associated mapping. A mapping $x \mapsto f_{x}$ on $A$ where $f_{x} \in \partial \phi(x)$ is called a support mapping of $\phi$ on $A$. It is clear that if $\phi$ is Gâteaux differentiable at $x \in A$ then $\partial \phi(x)$ is singleton and so the weak* upper semicontinuity of the subgradient mapping implies that every support mapping of $\phi$ is weak* continuous at $x$.

Lemma 1. If there exists a support mapping $x \mapsto f_{x}$ of $\phi$ on $A$ which is weak continuous (continuous) at $x \in A$ then $\phi$ is Gâteaux (Fréchet) differentiable at $x$.

Proof. Given $y \in X$ and $\lambda \in \mathbf{R}$ such that $x+\lambda y \in A$, and $f_{x} \in \partial \phi(x)$ and $f_{x+\lambda y} \in \partial \phi(x+\lambda y)$,

$$
f_{x}(\lambda y)<\phi(x+\lambda y)-\phi(x)<f_{x+\lambda_{y}}(\lambda y) .
$$

Therefore, for $\lambda>0$

$$
f_{x}(y)<\frac{\phi(x+\lambda y)-\phi(x)}{\lambda}<f_{x+\lambda y}(y)
$$

and the inequalities are reversed for $\lambda<0$. It follows that if there exists a support mapping $x \mapsto f_{x}$ which is weak* continuous (continuous) at $x$ then $\phi$ is Gâteaux (Fréchet) differentiable at $x$.

The following general property for continuous convex functions on a Banach space was given by Kenderov (1974).

Kenderov's THEOREM. For every continuous convex function $\phi$ on an open convex subset $A$ of a Banach space $(X,\|\cdot\|)$ there exists a dense $G_{8}$ subset of $A$ at each point $x$ of which, $\partial \phi(x)$ lies in the face of a sphere of $X^{*}$.

Proof. Kenderov showed that the function $\psi: A \rightarrow \mathbf{R}$ defined by

$$
\psi(x)=\inf \{\|f\|: f \in \partial \phi(x)\}
$$

is lower semi-continuous on $A$ and since $X$ is a Banach space then $\psi$ is continuous on a dense $G_{\delta}$ subset of $A$ (see Choquet (1969), p. 111). If $\psi$ is continuous at $x \in A$ and there exists an $f_{x} \in \partial \phi(x)$ such that

$$
\psi(x)<\left\|f_{x}\right\|
$$


then we can choose $y \in X,\|y\|=1$ such that

$$
\psi(x)<f_{x}(y)
$$

Since $\psi$ is continuous at $x$ there exists a $\lambda>0$ such that $\psi(x+\lambda y)<f_{x}(y)$ and an $f_{x+\lambda y} \in \partial \phi(x+\lambda y)$ such that

$$
f_{x+\lambda y}(y)<\psi(x+\lambda y)<f_{x}(y) .
$$

But this contradicts the fundamental inequalities given in Lemma 1.

The following significant result relating to the function $\psi$ was deduced by Fitzpatrick (1977).

Corollary. At any point $x \in A$ where $\psi$ is continuous, whenever $x_{n} \rightarrow x$ in $A$ then $\left\|f_{n}\right\| \rightarrow\|f\|$ for all $f_{n} \in \partial \phi\left(x_{n}\right)$ and $f \in \partial \phi(x)$.

We now examine the particular contribution of separability to our problem. It is well known that a Banach space with separable dual can be equivalently renormed to some advantage. Perhaps the most useful such renorming is with a Kadec-Klee norm. The following simplified version of this type of renorming which provides all that we require was given by Davis and Johnson (1973).

LEMMA 2. For a Banach space $X$ with separable dual $X^{*}$, there exists an equivalent norm $\|\cdot\|$ on $X$ with the property on $X^{*}$ that if the sequence $\left\{f_{n}\right\}$ is weak* convergent to $f$ and $\left\|f_{n}\right\| \rightarrow\|f\|$ then $\left\|f_{n}-f\right\| \rightarrow 0$.

Using Mazur's (1933) result on the Gâteaux differentiability of convex functions we are now in a position to prove the first stage of the characterisation theorem.

TheOREM (i) PART (a). A Banach space $X$ with separable dual $X^{*}$ is an Asplund space.

Proof. Consider $X$ renormed with a Kadec-Klee norm as given in Lemma 2. By Mazur's Theorem any given continuous convex function $\phi$ on an open convex $A \subseteq X$ is Gâteaux differentiable on a dense $G_{\delta}$ subset $A_{G}$ of $A$. By Kenderov's Theorem the function $\psi: A \rightarrow \mathbf{R}$ defined by

$$
\psi(x)=\inf \{\|f\|: f \in \partial \phi(x)\}
$$

is continuous on a dense $G_{\delta}$ subset $A_{\psi}$ of $A$. Consider $\phi$ on $A_{G} \cap A_{\psi}$ which is a dense $G_{\delta}$ subset of $A$. Now $\phi$ is Gâteaux differentiable on $A_{G} \cap A_{\psi}$ and so every support mapping of $\phi$ is weak* continuous on $A_{G} \cap A_{\psi}$. However, by Fitzpatrick's Corollary to Kenderov's Theorem, at each $x \in A_{G} \cap A_{\psi}$ whenever 
$x_{n} \rightarrow x$ in $A$ then $\left\|f_{n}\right\| \rightarrow\|f\|$ for all $f_{n} \in \partial \phi\left(x_{n}\right)$ and $f \in \partial \phi(x)$. Therefore, from the particular property of our Kadec-Klee norm we deduce that every support mapping of $\phi$ is continuous on $A_{G} \cap A_{\psi}$. From Lemma 1 we have that $\phi$ is Fréchet differentiable on $A_{G} \cap A_{\psi}$.

The proof of the converse result depends on a dual characterisation of Asplund spaces proposed initially by Namioka and Phelps (1975) and simplified significantly through an idea of Kenderov (1977). We give the proof of this theorem to show that a technique using approximate Fréchet differentiability produces a further simplification.

For a continuous convex function $\phi$ on an open convex subset $A$ of a normed linear space $(X,\|\cdot\|)$, given $\varepsilon>0$ we denote by $M_{\varepsilon}(\phi)$ the set of points $x \in A$ such that for some $\delta(\varepsilon, x)>0$

$$
\sup _{\substack{0<\lambda<\delta \\\|y\|=1}} \frac{\phi(x+\lambda y)+\phi(x-\lambda y)-2 \phi(x)}{\lambda}<\varepsilon .
$$

It can be shown that $\cap_{\varepsilon>0} M_{\varepsilon}(\phi)$ is the set of points of $A$ where $\phi$ is Fréchet differentiable.

The usefulness of approximate Fréchet differentiability depends on the following property.

LemMa 3. For a continuous convex function $\phi$ on an open convex subset $A$ of a normed linear space $(X,\|\cdot\|)$, given $\varepsilon>0$, the set $M_{e}(\phi)$ is open.

Proof. Consider $x \in M_{\varepsilon}(\phi)$. Since $\phi$ is continuous and convex it is locally Lipschitz; that is, there exists a $k>0$ anda $\delta^{\prime}>0$ such that

$$
|\phi(a)-\phi(b)|<k\|a-b\| \text { for all }\|a-x\|,\|b-x\|<\delta^{\prime} .
$$

Now there exists a $0<\delta(\varepsilon, x)<\delta^{\prime}$, and an $r>0$ such that

$$
\frac{\phi(x+\delta y)+\phi(x-\delta y)-2 \phi(x)}{\delta} \leqslant r<\varepsilon \text { for all }\|y\|=1 \text {. }
$$

Then

$$
\begin{aligned}
& \frac{\phi(z+\delta y)+\phi(z-\delta y)-2 \phi(z)}{\delta} \\
& \leqslant \frac{\phi(x+\delta y)+\phi(x-\delta y)-2 \phi(x)}{\delta}+\frac{1}{\delta}|\phi(z+\delta y)-\phi(x+\delta y)| \\
& \quad+\frac{1}{\delta}|\phi(z-\delta y)-\phi(x-\delta y)|+\frac{2}{\delta}|\phi(z)-\phi(x)| \\
& \leqslant r+\frac{4}{\delta} k\|z-x\| \quad \text { when }\|z-x\|<\delta^{\prime}-\delta \\
& <r+\varepsilon-r \text { when }\|z-x\|<\delta^{\prime \prime} \equiv \min \left\{\delta^{\prime}-\delta, \frac{\delta}{4 k}(\varepsilon-r)\right\} .
\end{aligned}
$$


Since $\phi$ is convex, the expression on the left decreases with $\delta$ and so $z \in M_{e}(\phi)$ for all $\|z-x\|<\delta^{\prime \prime}$.

The dual characterisation of Asplund spaces is expressed in terms of slices. Given a non-empty bounded set $C$ is a normed linear space $X$, if for any continuous linear functional $f$ on $X$ we write

$$
M(f, C) \equiv \sup \{f(x): x \in C\},
$$

then given $\alpha>0$ the subset of $C$

$$
S(C, f, \alpha) \equiv\{x \in C: f(x)>M(f, C)-\alpha\}
$$

is called a slice of $C$. Given a non-empty bounded set $C$ in $X^{*}$ for any $x \in X$ and $\alpha>0$ the slice

$$
S(C, x, \alpha) \equiv\{f \in C: f(x)>M(x, C)-\alpha\}
$$

is called a weak ${ }^{*}$ slice of $C$.

The Namioka-Phelps-Kenderov Theorem. A Banach space $(X,\|\cdot\|)$ is Asplund if and only if every bounded set in $X^{*}$ has weak* slices of arbitrarily small diameter.

Proof. Suppose that $C$ is a bounded set in $X^{*}$ and that there exists an $r>0$ such that every weak* slice of $C$ has diameter $>r$. Let $K$ denote the weak* closed convex hull of $C$. Then by the Banach-Alaoglu Theorem $K$ is weak* compact and every weak* slice of $K$ has diameter $>r$. We may assume that $0 \in K$ then the positive sublinear functional $p$ on $X$ defined by $p(x)=$ $\sup \{f(x): f \in K\} \equiv M(x, K)$ is continuous since $K$ is bounded. Given $x \in X$, since all slices of $K$ by $x$ have diameter $\geqslant r$ then for all $n \in N$

$$
\operatorname{diam}\left\{f \in X^{*}: f(x)>p(x)-\frac{r}{3 n}\right\} \geqslant r .
$$

So there exist sequences $\left\{f_{n}\right\},\left\{g_{n}\right\}$ in $K$ such that $f_{n}(x)>p(x)-r /(3 n)$ and $g_{n}(x)>p(x)-r /(3 n)$, and $\left\|f_{n}-g_{n}\right\|>r-1 / n$. Therefore there exists a sequence $\left\{y_{n}\right\},\left\|y_{n}\right\|=1$ such that $\left(f_{n}-g_{n}\right)\left(y_{n}\right)>r-1 / n$ and so

$$
\begin{aligned}
p\left(x+\frac{1}{n} y_{n}\right)+p(x & \left.-\frac{1}{n} y_{n}\right)-2 p(x) \\
& \geqslant f_{n}\left(x+\frac{1}{n} y_{n}\right)+g_{n}\left(x-\frac{1}{n} y_{n}\right)-2\left(f_{n}+g_{n}\right)(x)-\frac{2 r}{3 n} \\
& =\frac{1}{n}\left(f_{n}-g_{n}\right)\left(y_{n}\right)-\frac{2 r}{3 n} \\
& >\frac{r}{3 n}-\frac{1}{n^{2}} .
\end{aligned}
$$


This implies that $p$ is not Fréchet differentiable at $x$ and so is not Fréchet differentiable at any point of $X$.

Conversely, consider a continuous convex function $\phi$ on an open convex $A \subset X$. Since the subgradient mapping is locally bounded, given $x \in A$ there exists a neighbourhood of $U$ of $x$ such that $\partial \phi(U)$ is bounded. Given $\varepsilon>0$ there exists a $z \in X$ and an $\alpha>0$ such that the weak* slice $S(\partial \phi(U), z, \alpha)$ has diameter $<\varepsilon$. If $f \in S(\partial \phi(U), z, \alpha)$ then $f \in \phi\left(x_{1}\right)$ for some $x_{1} \in U$. Now $x_{0} \equiv x_{1}+r z \in U$ for sufficiently small $r>0$. For $g \in \partial \phi\left(x_{0}\right)$

$$
\begin{aligned}
& g\left(x_{1}-x_{0}\right)<\phi\left(x_{1}\right)-\phi\left(x_{0}\right), \\
& f\left(x_{0}-x_{1}\right)<\phi\left(x_{0}\right)-\phi\left(x_{1}\right) .
\end{aligned}
$$

Adding we have

$$
0<(g-f)\left(x_{0}-x_{1}\right)=r(g-f)(z)
$$

so that

$$
g(z)>f(z)>M(z, \partial \phi(U))-\alpha
$$

which implies that $\partial \phi\left(x_{0}\right) \subseteq S(\partial \phi(U) z, \alpha)$. Since $S(\partial \phi(U) z, \alpha)$ is a relative weak $^{*}$ open subset of $\partial \phi(U)$ we have from the weak* upper semi-continuity of the subgradient mapping that there exists a $\delta\left(x_{0}\right)>0$ such that for any $y \in X$, $\|y\|=1, \partial \phi\left(x_{0}+\lambda y\right)$ and $\partial \phi\left(x_{0}-\lambda y\right) \subseteq S(\partial \phi(U) z, \alpha)$ for $0<\lambda<\delta$. Therefore, for any $f_{x_{0}+\lambda y} \in \partial \phi\left(x_{0}+\lambda y\right)$ and $f_{x_{0}-\lambda y} \in \partial \phi\left(x_{0}-\lambda y\right)$ we have

$$
\begin{aligned}
\frac{\phi\left(x_{0}+\lambda y\right)+\phi\left(x_{0}-\lambda y\right)-2 \phi\left(x_{0}\right)}{\lambda} & <\left(f_{x_{0}+\lambda y}-f_{x_{0}-\lambda_{y}}\right)(y) \\
& <\left\|f_{x_{0}+\lambda y}-f_{x_{0}-\lambda y}\right\| \\
& <\varepsilon \text { for } 0<\lambda<\delta ;
\end{aligned}
$$

that is, $x_{0} \in M_{\varepsilon}(\phi)$. We have then that every neighbourhood $U$ of $x$ contains a point of $M_{\varepsilon}(\phi)$; that is, for each $\varepsilon>0, M_{\varepsilon}(\phi)$ is dense in $A$. However, $A$ is a Baire space so $\cap_{n \in N} M_{1 / n}(\phi)$, the set of points where $\phi$ is Fréchet differentiable on $A$, is a dense $G_{\delta}$ subset of $A$.

We now complete the characterisation theorem for separable spaces following the proof of Namioka and Phelps (1975).

Theorem (i) PART (b). A separable Asplund space $X$ has separable dual $X^{*}$.

Proof. If $X^{*}$ is not separable there exists an $r>0$ and an uncountable subset $C$ of the unit ball $B\left(X^{*}\right)$ such that $\|f-g\|>r$ for all $f, g \in C$. Since $X$ is separable the weak* topology on $B\left(X^{*}\right)$ is metrisable so it is second countable. Therefore, $C$ has at most countably many points which are not weak* condensation points. We may assume that these points have been deleted from $C$. Now 
by the Namioka-Phelps-Kenderov Theorem there exists a weak* slice of $C$ with diameter $<r$. This weak* slice is a relatively weak* open subset of $C$. But no point of $C$ is weak* isolated so there exist distinct $f, g \in C$ in this slice. However, this contradicts $\|f-g\|>r$.

\section{The characterisation theorem for the general case}

We now proceed to show that the Asplund property is separably determined. The first part of this result was obtained by Namioka and Stegall by dual methods and the proof is outlined in Diestel and Uhl (1977), page 213. The following more direct proof using the technique of approximate Fréchet differentiability is due to D. A. Gregory.

Theorem (ii) PART (a). A Banach space $X$ is Asplund if every closed separable subspace of $X$ is Asplund.

Proof. Let $\phi$ be a continuous convex function on an open convex $A \subseteq X$ where the set of points of Fréchet differentiability is not dense in $A$. We show that there exists a closed separable subspace $Y$ of $X$ such that $A \cap Y \neq \varnothing$ and the set of points of Fréchet differentiability of $\left.\phi\right|_{Y}$ is not dense in $A \cap Y$. Since $A$ is a Baire space for some $r>0, M_{r}(\phi)$ is not dense in $A$ so there exists an open set $G \subseteq A \backslash M_{r}(\phi)$. We define inductively an increasing sequence of separable subspaces $\left\{Y^{n}\right\}$ of $X$. First choose $y^{1} \in G$ and a sequence $\left\{z_{k}^{1}\right\}$, $\left\|z_{k}^{1}\right\|=1$ such that

$$
\sup _{k \in \mathbb{N}} \frac{\phi\left(y^{1}+\lambda z_{k}^{1}\right)+\phi\left(y^{1}-\lambda z_{k}^{1}\right)-2 \phi\left(y^{1}\right)}{\lambda}>r
$$

for all $\lambda>0$, and let $Y^{1}$ denote the closed subspace spanned by $y^{1}$ and $\left\{z_{k}^{1}\right\}$. Now given a subspace $Y^{n}$ define a subspace $Y^{n+1}$ as follows. Choose a countable dense set $\left\{y_{m}^{n}: m \in \mathbf{N}\right\}$ in $Y^{n} \cap G$ and for each $m \in \mathbf{N}$ choose a sequence $\left\{z_{k}^{m}\right\},\left\|z_{k}^{m}\right\|=1$ such that

$$
\sup _{k \in N} \frac{\phi\left(y_{m}^{n}+\lambda z_{k}^{m}\right)+\phi\left(y_{m}^{n}-\lambda z_{k}^{m}\right)-2 \phi\left(y_{m}^{n}\right)}{\lambda}>r
$$

for all $\lambda>0$. Then let $Y^{n+1}$ denote the closed subspace spanned by $Y^{n}$ and $\left\{y_{m}^{n}, z_{k}^{m}: m, k \in \mathrm{N}\right\}$. Writing $Y \equiv \overline{\cup_{n \in N} Y^{n}}$ we have that $\left\{y_{m}^{n}: n, m \in \mathbf{N}\right\}$ is dense in $Y \cap G$. By the construction, $\left\{y_{m}^{n}: n, m \in \mathbf{N}\right\} \subseteq(A \cap Y) \backslash M_{r}\left(\left.\phi\right|_{Y}\right)$ and since $(A \cap Y) \backslash M_{r}\left(\left.\phi\right|_{Y}\right)$ is a closed subset of $A \cap Y$ we have $Y \cap G \subseteq$ $(A \cap Y) \backslash M_{r}\left(\left.\phi\right|_{Y}\right)$. But then $\left.\phi\right|_{Y}$ is not Fréchet differentiable on the relatively open set $Y \cap G$. 
The convese result is a special case of the following more general theorem of Namioka and Phelps (1975) and we include the proof for the sake of completeness.

TheOREM Which INCLUdes (ii) PART (b). A closed subspace $M$ of an Asplund space $X$ is an Asplund space.

Proof. Let $C$ be a bounded set in $M^{*}$ and $K$ be the weak* closed convex hull of $C$. We show that $K$ has weak* slices of arbitrarily small diameter and consequently that $C$ has weak* slices of arbitrarily small diameter.

Consider the quotient mapping $Q: X^{*} \rightarrow X^{*} / M^{\perp}=M^{*}$ defined by $Q(f)=f$ $+M^{\perp}$. Since $q$ is an open mapping, $Q\left(B\left(X^{*}\right)\right)$ is a neighbourhood of 0 in $M^{*}$. Since $K$ is bounded in $M^{*}$ there exists $\lambda>0$ such that $Q\left(\lambda B\left(X^{*}\right)\right)=\lambda Q\left(B\left(X^{*}\right)\right)$ $\supseteq K$. Since $Q$ is continuous when $X^{*}$ has the weak* topology and $M^{*}$ has the weak ${ }^{*}$ topology, the convex set $B \equiv \lambda B\left(X^{*}\right) \cap Q^{-1}(K)$ is weak ${ }^{*}$ compact in $X^{*}$ and $Q(B)=K$. Consider the family of all weak* compact convex sets $B_{\alpha}$ in $X^{*}$ such that $Q\left(B_{\alpha}\right)=K$, partially ordered by set inclusion. By Zorn's Lemma there exists a minimal set $B_{0}$ in this family. Now since $X$ is Asplund, given $\varepsilon>0$ there exists a weak* slice of $B_{0}$,

$$
S\left(B_{0}, x, \alpha\right) \equiv\left\{f \in B_{0}: f(x)>M\left(x, B_{0}\right)-\alpha\right\}
$$

of diameter $\leqslant \varepsilon$. Then $K_{0} \equiv Q\left(B_{0} \backslash S\left(B_{0}, x, \alpha\right)\right)$ is a weak* compact convex set which by the minimality of $B_{0}$ is properly contained in $K$. For any $f_{1}, f_{2} \in$ $K \backslash K_{0}$ there exist $g_{1}, g_{2} \in S\left(B_{0}, x, \alpha\right)$ such that $Q\left(g_{1}\right)=f_{1}$ and $Q\left(g_{2}\right)=f_{2}$ and

$$
\left\|f_{1}-f_{2}\right\|=\left\|Q\left(g_{1}-g_{2}\right)\right\|<\left\|g_{1}-g_{2}\right\|<\varepsilon .
$$

So $\operatorname{diam}\left(K \backslash K_{0}\right) \leqslant \varepsilon$. Since $K_{0}$ is a weak* compact convex set we have by the Separation Theorem that there exists a weak ${ }^{*}$ slice of $K$ which does not intersect $K_{0}$ and so has diameter $\leqslant \varepsilon$. We conclude from the Namioka-Phelps-Kenderov Theorem that $M$ is Asplund.

\section{The 'smooth bump function' condition}

For a Banach space, Ekeland and Lebourg (1976) gave what is called 'the smooth bump function' condition to guarantee differentiability properties for a large class of functions which include the continuous convex functions. This was done by using a generalisation of the Bishop-Phelps Theorem devised by Ekeland (1974). We present a proof a little simpler than that offered by Ekeland (1979) that the smooth bump function condition implies that a Banach space has the Asplund property. 
Ekeland's form of THE Bishop-Phelps Theorem. Let $(X, d)$ be a complete metric space and $\psi$ a proper extended real lower semi-continuous function on $X$ bounded from below. Given $\varepsilon>0$, there exists an $x \in X$ such that

$$
\psi(x)<\inf \{\psi(z): z \in X\}+\varepsilon \text { and } \psi(z) \geqslant \psi(x)-\varepsilon d(z, x) \text { for all } z \in X \text {. }
$$

The EKeland-Lebourg Theorem. If a Banach space $(X,\|\cdot\|)$ admits a real Fréchet differentiable function $\theta$ on $X$ with bounded non-empty support then $X$ is an Asplund space.

Proof. Consider a continuous convex function $\phi$ on an open convex $A \subseteq X$. Given $a \in A$, there exists an $r>0$ such that $B[a ; r] \subseteq A$ and $\phi$ is bounded on $B[a ; r]$. Consider $\phi$ scaled and translated so that $\operatorname{supp} \theta \subseteq B[a ; r]$. Now $1 / \theta$ is Fréchet differentiable on dom $1 / \theta \subseteq B[a ; r]$.

For $1 / \theta-\phi$ on $B[a ; r]$, given $\varepsilon>0$ there exists an $x \in B[a ; r]$ such that

$$
\begin{aligned}
& \left(\frac{1}{\theta}-\phi\right)(x) \leqslant \inf \left\{\left(\frac{1}{\theta}-\phi\right)(z): z \in B[a ; r]\right\}+\varepsilon \text { and } \\
& \left(\frac{1}{\theta}-\phi\right)(z) \geqslant\left(\frac{1}{\theta}-\phi\right)(x)-\varepsilon\|z-x\| \text { for all } z \in B[a ; r] .
\end{aligned}
$$

Since $1 / \theta(z)$ is finite for some $z \in B[a ; r]$ and $\phi(z)$ is bounded on $B[a ; r]$, we deduce that $1 / \theta(x)$ is finite; that is, $x \in \operatorname{dom} 1 / \theta$. Now $1 / \theta$ is Fréchet differentiable at $x$ so there exists a $\delta>0$ such that $B(x ; \delta) \subseteq$ dom $1 / \theta$ and

$$
\frac{1}{\theta}(z)-\frac{1}{\theta}(x)-\left(\frac{1}{\theta}\right)^{\prime}(z-x) \leqslant \varepsilon\|z-x\| \text { for all }\|z-x\|<\delta .
$$

Adding (i) and (ii) we have

$$
\phi(z)-\phi(x)-\left(\frac{1}{\theta}\right)^{\prime}(z-x) \leqslant 2 \varepsilon\|z-x\| \text { for all }\|z-x\|<\delta .
$$

Writing $z \equiv x+\lambda y$ where $\|y\|=1$, then

$$
\phi(x+\lambda y)-\phi(x)-\lambda\left(\frac{1}{\theta}\right)^{\prime}(y) \leqslant 2 \varepsilon|\lambda| \text { for all }|\lambda|<\delta \text { and }\|y\|=1 .
$$

Since $\phi$ is convex,

$$
0 \leqslant \frac{\phi(x+\lambda y)+\phi(x-\lambda y)-2 \phi(x)}{\lambda} \leqslant 4 \varepsilon \text { for all } 0<\lambda<\delta .
$$

But this implies that $x \in M_{5 \varepsilon}(\phi)$. We deduce that for every $\varepsilon>0, M_{e}(\phi)$ is dense in $A$. However, $A$ is a Baire space so $\bigcap_{n \in N} M_{1 / n}(\phi)$ the set of points where $\phi$ is Fréchet differentiable on $A$ is a dense $G_{\delta}$ subset of $A$.

The following well-known result can be deduced quite simply. 
COROLlary. If a Banach space $X$ can be equivalently renormed to have a norm Fréchet differentiable on $X \backslash\{0\}$ then $X$ is an Asplund space.

Proof. Consider a differentiable function $g$ on $\mathbf{R}$ with bounded non-empty support. If $X$ is normed with norm $\|\cdot\|$ Fréchet differentiable on $X \backslash\{0\}$ then $\theta=g \circ\|\cdot\|^{2}$ is a 'smooth bump function' for the Ekeland-Lebourg Theorem.

I am indebted to the referee for pointing out that another proof of the Ekeland-Lebourg Theorem follows from Theorem (i) part (a) and the result of Leach and Whitfield (1972) that if a Banach space $X$ admits a 'smooth bump function' then for all subspaces $Y$ of $X$, dens $Y=\operatorname{dens} Y^{*}$.

\section{References}

E. Asplund (1968), 'Fréchet differentiability of convex functions', Acta Math. 121, 31-47.

G. Choquet (1969), Lectures on analysis, vol. 1 (Benjamin, New York).

W. J. Davis and W. B. Johnson (1973), 'A renorming of nonreflexive Banach spaces', Proc. Amer. Math. Soc. 37, 486-487.

J. Diestel and J. J. Uhl (1977), Vector measures, Math. Surveys No. 15 (Amer. Math. Soc., Providence, R. I.).

I. Ekeland (1974), 'On the variational principle', J. Math. Anal. Appl. 47, 324-353.

I. Ekeland (1979), 'Nonconvex minimization problems', Bull. Amer. Math. Soc. (N.S.) 1, 443-474.

I. Ekeland and G. Lebourg (1976), 'Generic Fréchet-differentiability and perturbed optimisation problems in Banach spaces', Trans. Amer. Math. Soc. 224, 193-216.

S. P. Fitzpatrick (1977), 'Continuity of nonlinear monotone operators', Proc. Amer. Math. Soc. 62, $111-116$.

P. S. Kenderov (1974), 'The set-valued monotone mappings are almost everywhere single-valued', $C$. R. Acad. Bulgare Sci. 27, 1173-1175.

P. S. Kenderov (1977), 'Monotone operators in Asplund spaces', C. R. Acad. Bulgare Sci. 30, 963-964.

E. B. Leach and J. H. M. Whitfield (1972), 'Differentiable functions and rough norms on Banach spaces', Proc. Amer. Math. Soc. 33, 120-126.

S. Mazur (1933), 'Uber konvexe Mengen in linearen normierten Räumen', Studia Math. 4, 70-84.

I. Namioka and R. R. Phelps (1975), 'Banach spaces which are Asplund spaces', Duke Math. J. 42, 735-749.

R. R. Phelps (1978), Differentiability of convex functions on Banach spaces (Lectures Notes, University of London).

C. Stegall (1975), 'The Radon-Nikodým property in conjugate Banach spaces', Trans. Amer. Math. Soc. 206, 213-223.

C. Stegall (1978), The duality between Asplund spaces and spaces with the Radon-Nikodym property', Israel J. Math. 29, 408-412.

University of Newcastle

Newcastle, N.S.W. 2308

Australia 